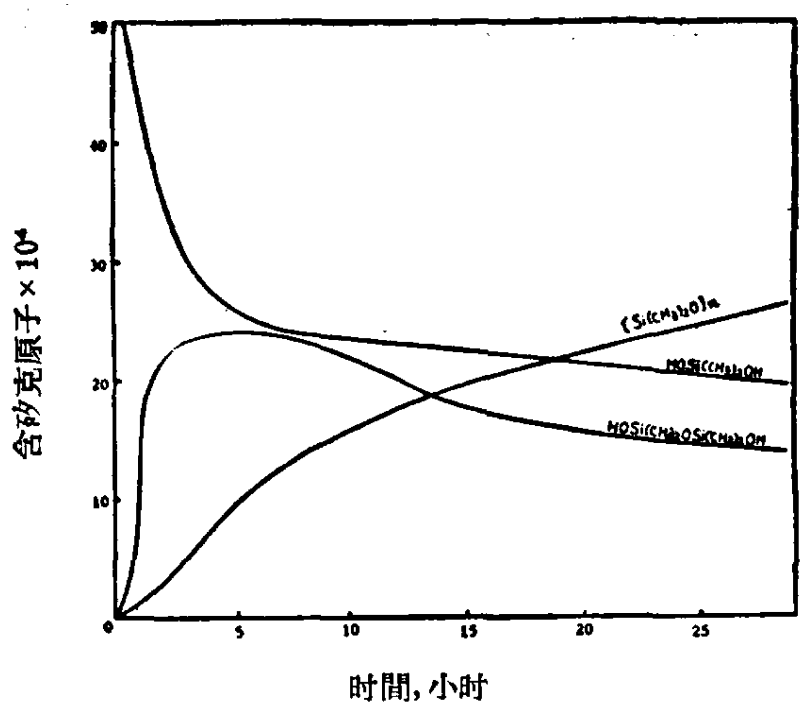

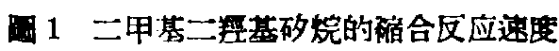

差基二矽氧烷之下。但这試驗結果倹明二甲基二差基 矽烷的濃度不但沿有迅速下降，而且一直維持在对称 四甲基二差基二矽氧烷之上。这显示着二甲基二差基 矽烷与对称四甲基二垟基二矽䂇烷之間有个平衡状 态。这平衡常数 $\left(18^{\circ} \mathrm{C}\right)$ 是

$$
\frac{\left(\mathrm{HOSi}\left(\mathrm{CH}_{3}\right)_{2} \mathrm{OSi}\left(\mathrm{CH}_{3}\right)_{2} \mathrm{OH}\right]}{\left[\mathrm{HOSi}\left(\mathrm{CH}_{3}\right)_{2} \mathrm{OH}\right]^{2}}=1.95
$$

二甲基二逕基矽烷公認为是十分不隐定的[2]，很容易 变成对称四甲基二差基二矽氧烷和高聚物。所以这本 微的存在是很出人意料之外的。

为了明确这个問題，我們另从对称四甲基二差基 二矽篻烷出被, 用同样的实驗方法进行动力学研究(温 度改为 $30^{\circ} \mathrm{C}$ )，結果(見圆 2 ) 証明了对称四甲基二跮

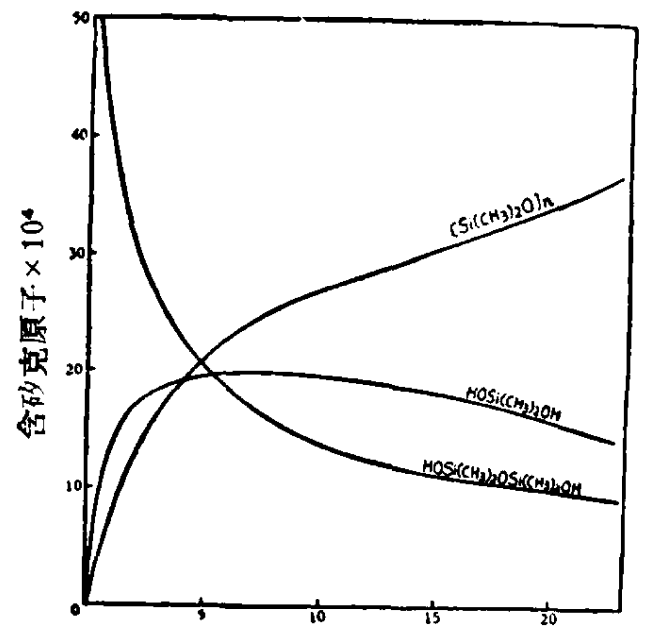

时間, 小时

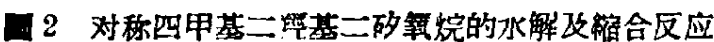

基二矽氧烷确能在酸性介質中，一面进行縮合反应， 一面水解为二甲基二差基矽烷，两物間的平衡常数 $\left(30^{\circ} \mathrm{C}\right)$ 为 2.11 。

.林劣安林 一

(中山大学化学禾)（中国科学院化学研究所） 1957 年 9 月 18 日
[1] Grubb, J. Am. Chem. Soc., 76,3408(1954).

[2] Hyde, J. Am. Chem. Soc., 75, 2166(1953);

Kantor, J. Am. Chem. Soc., 75, 2712(1953).

\section{5-眿基-8-坘基7奎啉的合成及其对一 些金屬离子的选擇性和灵敏度}

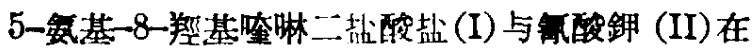
水溶液中作用后，得到一种新的化合物- - 5-豚悲-8帮基腟晽盐酸盐(III)，熔点 $246-247^{\circ}$ (分解)(自含有 少量盐酸之甲醇一水或乙醇一水中重結晶)，为金璜色 菱形結晶。

分析: $\mathrm{C}_{10} \mathrm{H}_{10} \mathrm{~N}_{3} \mathrm{O}_{2} \mathrm{Cl}$

$$
\begin{aligned}
& \text { 計算徝\%： } \mathrm{Cl}, 14.80 \\
& \text { 实駔做只: Cl, } 15.09
\end{aligned}
$$

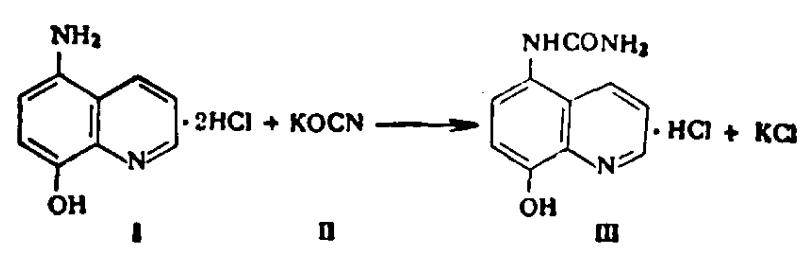

\begin{tabular}{|c|c|c|c|}
\hline 金曆离子 & 沉资頝色 & pL値 & $\begin{array}{l}\text { 想常于 } \\
\text { 党/升 }\end{array}$ \\
\hline $\mathrm{Cu}+2$ & 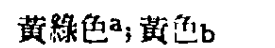 & $4.69-4.80$ & $0.635-0.508$ \\
\hline $\mathrm{Zn}+2$ & 黄色 & $3.10-3.40$ & $26.2-13.1$ \\
\hline $\mathrm{Cd}+2$ & 韁色 & $1.40-2.44$ & $2250-2020$ \\
\hline $\mathrm{Hg}+2$ & 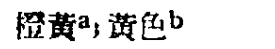 & $3.80-3.93$ & $16.1--12.0$ \\
\hline $\mathrm{Mn}+2$ & 深黃包 & $3.10-3.40$ & $22.0--11.0$ \\
\hline $\mathrm{Co}+2$ & 黃色 & $3.10-3.40$ & $23.6-11.8$ \\
\hline $\mathrm{Ni}+2$ & 读色 & $3.10-3.40$ & $23.5--11.7$ \\
\hline $\mathrm{WO}_{4}=$ & 黃色 & $1.40-2.44$ & $4960-4460$ \\
\hline $\mathrm{Pb}^{+2}$ & 责色 & $2.69-2.80$ & $207-166$ \\
\hline $\mathrm{Ce}^{+3}$ & 黄色 & $3.74--3.92$ & $8.41-4.81$ \\
\hline $\mathrm{Mg}^{+2}$ & 没有沉谈 & & \\
\hline $\mathrm{Ca}^{+2}$ & 没有沉俒 & & \\
\hline $\mathrm{Sr}+2$ & 没有沉腚 & & \\
\hline $\mathrm{Ba}+2$ & 没有沉腚 & & \\
\hline $\mathrm{Fe}^{+3}$ & 伍䅑冲溶液中不加 & & \\
\hline $\mathrm{Al}+3$ & 鼠氧化物沉腚出来 & & \\
\hline $\mathrm{Cr}+3$ & 沒有沉栬 & & \\
\hline $\mathrm{McO}_{4}=$ & 没有沉淡 & & \\
\hline $\mathrm{La}^{+3}$ & 沒有沉腚 & & \\
\hline $\mathrm{Th}^{+4}$ & 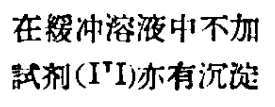 & & \\
\hline $\mathrm{SeO}_{3}=$ & 没有沉栬 & & \\
\hline
\end{tabular}

表 1

$\mathrm{pH}=5.30$

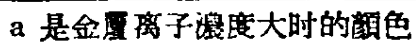

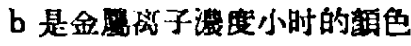


搵酸盐(III)的水溶洨以碳酸鉡处理后，得到游离

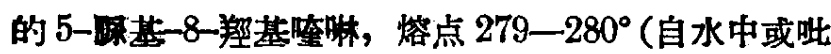

定中重結晶)，为灰白色針状結晶。

分析: $\mathrm{C}_{10} \mathrm{H}_{2} \mathrm{~N}_{3} \mathrm{O}_{2}$

計算值\%: $\mathrm{C}, 59.00, \mathrm{H}, 4.46 ; \mathrm{N}, 20.68$

实驗值\%: C, $59.32 ; \mathrm{H}, 4.50 ; \mathrm{N}, 20.45,20.57$

$\mathrm{Mn}^{+2}, \mathrm{WO}_{4}=, \mathrm{Pb}^{+2}, \mathrm{Ce}^{+3}$

等有沉腚, $\mathrm{Fe}^{+3}, \mathrm{Al}^{+3}$,

$\mathrm{Th}+4$ 空白亦有沉淀, 为水解 所得的昰氧化物。本試刺 (III) 与那些生成沉淀的金 闽离子的灵敏度用 $\mathrm{pL}$ 值表 示, $\mathrm{pL}$ 值是 Irving[1]等建 議抹为 Hollingshead[2]所

\begin{tabular}{|c|c|c|c|c|c|c|c|c|c|}
\hline \multirow{2}{*}{ 試 } & \multicolumn{9}{|c|}{ 試剂对金屡离子的灵政度（用 $\mathrm{pL}$ 值表示） } \\
\hline & $\mathrm{Cu}^{+2}$ & $\mathrm{Zn}+2$ & $\mathrm{Cd}+2$ & $\mathrm{Hg}^{+2}$ & $\mathrm{Co}^{+2}$ & $\mathrm{Ni}+2$ & $\mathrm{~Pb}^{+2}$ & $\mathrm{Ce}^{+3}$ & $\mathrm{Mg}^{+2}$ \\
\hline 5-服基-8-塑基埕啉 & 4.69 & 3.10 & 1.40 & 3.80 & 3.10 & 3.10 & 2.69 & 3.74 & 没有沉 \\
\hline $\begin{array}{l}\text { 5-甲基-7-亚的基-8- } \\
\text { 羥基唋物 }\end{array}$ & 3.24 & 5.34 & 4.84 & 5.54 & 4.84 & 4.84 & 4.84 & 3.24 & 4.24 \\
\hline
\end{tabular}

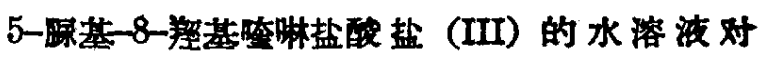
$\mathrm{Mg}^{+2}, \mathrm{Ca}^{+2}, \mathrm{Sr}^{+2}, \mathrm{Ba}^{+2}, \mathrm{Zn}{ }^{+2}, \mathrm{Cd}^{+2}, \mathrm{Hg}^{+2}, \mathrm{Cu}^{+2}$, $\mathrm{Fe}^{+3}, \mathrm{Co}^{+2}, \mathrm{Ni}^{+2}, \mathrm{Al}^{+3}, \mathrm{Cr}^{+3}, \mathrm{Mn}^{+2}, \mathrm{~Pb}^{+2}$, $\mathrm{MoO}_{4}=, \mathrm{WO}_{4}=, \mathrm{Th}^{+4}, \mathrm{Ce}+3, \mathrm{La}^{+3}, \mathrm{SeO}_{3}=$ 等 21 种金 畋离子作用，在 $\mathrm{pH}=5.30$ 的緩冲溶液 (Na Ac-HAC 水溶液) 中, 仅 $\mathrm{Zn}+2, \mathrm{Cd}^{+2}, \mathrm{Hg}^{+2}, \mathrm{Cu}^{+2}, \mathrm{Co}^{+2}, \mathrm{Ni}^{+2}$,

表 3
表 $2 \mathrm{pH}=2.60$

\begin{tabular}{|c|c|c|c|}
\hline 金擂子 & 沉 淀 顠 色 & $\mathrm{pL}$ 值 & $\begin{array}{l}\text { 想当党/升 } \\
\text { 管 }\end{array}$ \\
\hline $\mathrm{Cu}+2$ & 黄色 & $4.55-4.62$ & $0.890-0.763$ \\
\hline $\mathrm{Zn}+2$ & 没有沉煏 & & \\
\hline $\mathrm{Cd}+\mathbf{2}$ & 没有沉谈 & & \\
\hline $\mathrm{Hg}^{+2}$ & 沟有沉柁 & & \\
\hline $\mathrm{Mn}+2$ & 沒有沉栬 & & \\
\hline $\mathrm{Co}+2$ & 没有沉栬 & & \\
\hline $\mathrm{Ni}+2$ & 没有沉淀 & & \\
\hline $\mathrm{WO}_{4}=$ & 没有沉腚 & & \\
\hline $\mathrm{Pb}+2$ & 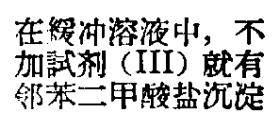 & & \\
\hline $\mathrm{Ce}^{+3}$ & 沒有沉淀 & & \\
\hline $\mathrm{Mg}^{+2}$ & 没有沉栬 & & \\
\hline $\mathrm{Ca}_{\mathrm{a}}+2$ & 没有沉资 & & \\
\hline $\mathrm{Sr}+2$ & 没有沉栬 & & \\
\hline $\mathrm{Ba}+2$ & 沒有沉淀 & & \\
\hline $\mathrm{Fe}+3$ & 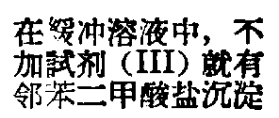 & & \\
\hline $\mathrm{Al}^{+3}$ & 没有沉资 & & \\
\hline $\mathrm{Cr}+3$ & 没有沉资 & & \\
\hline $\mathrm{MoO}_{4}=$ & 没有沉淀 & & \\
\hline $\mathrm{La}+3$ & 没有沉栬 & & \\
\hline $\mathrm{Th}^{+4}$ & 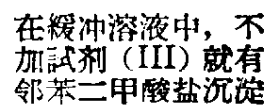 & & \\
\hline $\mathrm{SeO}_{3}=$ & 没有沉腚 & & \\
\hline
\end{tabular}

沿用。此种标衭灵敏度的方法是: 在室温 (約 $20^{\circ}$ ) 时，与試剂作用得到沉淀所需最少量金屬离子濃度 (克当量数/升)的 $-\log$ 值。測定的方法是: 在一系列 相同量的試剂中加入繼續减少合量的金屬离子溶液遖 到岡能鸴察出沉淀时为止。其結果如表 1 (試 剂 III 为 $0.02 \mathrm{M}$ 的水溶液)。

在 $\mathrm{pH}=2.60$ 的緩冲溶液 (邻苯二甲酸氠鈝一稀盐
酸溶液)中, 本試剂 (III) 对上迅 21 种金屬离子实騟 的結果表明: 仅 $\mathrm{Cu}+2$ 有沉腚, 其灵敏度用 $\mathrm{pL}$ 值表示 如表 2 。 $\mathrm{Th}^{+4}, \mathrm{Fe}^{+3}, \mathrm{~Pb}^{+2}$ 空白有沉淀, 可能是其 邻苯二甲酸盐。

上迅实驗結果和 R. G.W. Hollingshead[2]在相 同条件下 $(\mathrm{pH}=5.30$ ) 用 5-甲基-7-亚砣基-8-帮基啅 晽为試剂时所得的結果比較如表 3 。

本試剂相当稳定，不易被氧化，同时对金屈离子 的选擇性份佳，且由于其对 $\mathrm{Cu}+2$ 的灵敏度甚高，而 对于其他金屬离子的灵敏度較低, 因此, 5-豚基-8差基鈤晽或許可能作为一种分析銅的有机試剂。

\section{陈淑英 黄文鬿 \\ (兰州大学化学禾) \\ 1957 年 9 月 20 日}

[1] H. Irving, E. J. Butler and M. F. Ring, J. Chem. Soc., $1949,1489$.

[ 2 ] R. G. W. Hollingshead, Anal. Chimica Acta, 12, 201(1955).

\section{两株擲孢酵母所生 Biotin 的鍳定}

在我們測驗一种䤃母菌 (2.346 号 Saccharomyces)的单倍体及双倍体所需要生长素是否一致时, 見到一个小菌落能生多量的生长素。經过分离后, 得 到四种菌：两株撽抱酵母 (Sporobolomyces)，一株 要菌及一株普通酵母, 前三者均能合成生长素(圖 1)。

我們近来用生长圖形法 (Auxanographic method) 研究䐗母生长素的經驗, 訅为这个方法比我們， 以前所用的重量法[1]、計数法[2]、發胠法[3]、美藍还 原法[4]等簡便得多; 已經用这个方法測驗惟不少腾时 